\title{
Filtro Hodrick-Prescott Aplicado à Análise de Sinais Elétricos com Distúrbios de Qualidade de Energia Elétrica
}

\author{
Danielle A. Fortunato*, Márcio W. Santana*, Jader Bosco Gomes* \\ *Centro Federal De Educação tecnológica De Minas Gerais - \\ CEFET-MG, Nepomuceno, Brasil \\ Tel: (35) 3861- 4500 \\ E-mails:danielle.afortunato@gmail.com, \\ marciosantana@cefetmg.br,jader@cefetmg.br
}

\begin{abstract}
Power quality disturbances can occur in various parts of the power system causing financial losses. Therefore, automatic detection of these disturbances with high performance is crucial. This work aims to evaluate the performance of the Hodrick-Prescott filter in the pre-processing stage of an Power Quality monitoring system. The proposal is to detect the presence of Power Quality disturbances in electrical power systems using an Artifical Neural Network, where its architecture is of the Multilayer Perceptron type. In the preprocessing step of the input data, the Hodrick-Prescott filter divides the input signal into a trend component and a cyclic component for the extraction of variables indicative of the presence of disturbances in conjunction with the Fisher Discriminat Ratio that is used for parameter selection to be analyzed by Artifical Neural Network. Detection of disturbances was achieved with mean performance of $99 \%$.
\end{abstract}

Resumo: Distúrbios de qualidade de energia elétrica podem ocorrer em várias partes do sistema de energia causando prejuízos financeiros. Por isso, é crucial a detecção automática destes distúrbios com alto desempenho. Este trabalho tem por objetivo avaliar o desempenho do filtro Hodrick-Prescott na etapa de pré-processamento de um sistema de monitoramento de Qualidade de Energia Elétrica. A proposta é detectar a presença de distúrbios de Qualidade de Energia Elétrica em sistemas elétricos de potência utilizando uma Rede Neural Artificial, onde sua arquitetura é do tipo Multilayer Perceptron. Na etapa de pré-processamento dos dados de entrada o filtro Hodrick-Prescott divide o sinal de entrada em componente de tendência e componente cíclica, para extração de variáveis indicadoras da presença de distúrbios em conjunto com o Discriminante Linear de Fisher que é utilizado para seleção de parâmetros a serem analisados pela Rede Neural. A detecção de distúrbios foi alcançada com desempenho médio de $99 \%$.

Keywords: Hodrick-Prescott Filter; Fisher Discriminat Ratio; Disturbances; Power Qualiy; Artifical Neural Network.

Palavras-chaves: Filtro Hodrick-Prescott; Discriminante Linear de Fisher; Distúrbios; Qualidade de Energia Elétrica; Redes Neurais Artificiais.

\section{INTRODUÇÃO}

Atualmente a qualidade da energia elétrica é um requisito de grande importância para as concessionárias de energia, pois está diretamente relacionada à competitividade em praticamente todos os setores industriais e de serviços. Sobretudo nas últimas duas décadas aconteceram várias mudanças no setor elétrico, tais como a alteração da natureza das cargas, diversificação dos tipos de cargas, e o surgimento de novas tecnologias de geração e inserção de energia na rede elétrica. Assim, estas alterações afetaram de forma negativa a Qualidade da Energia Elétrica (QEE) promovendo o aumento dos estudos relacionados a essa área e a busca por qualidade passou a ser preocupação não apenas de órgão reguladores, mas também de consumidores e agentes diversificados do setor elétrico nacional. Um exemplo que evidencia tais mudanças citadas é o uso de Geração Distribuída (GD), onde a geração elétrica realizada junto ou próxima dos consumidores independente da potência, tecnologia e fonte de energia (INEE, 2011; Di Santo, et al. 2015; El-Hawary, 2014).

O objetivo deste trabalho é avaliar o desempenho do filtro Hodrick-Prescott na etapa de pré-processamento de um sistema de monitoramento de Qualidade de Energia Elétrica, como ferramenta para extração de características indicadoras de presença de distúrbio. A Rede Neural Artificial do tipo MLP (Multilayer Perceptron) é utilizada para realizar a detecção automática de distúrbios de QEE, os quais podem ocorrer em várias partes do sistema de energia causando prejuízos financeiros tanto às concessionárias fornecedoras de energia quanto aos seus consumidores. 
A detecção deve acontecer em modo off-line, ou seja, os dados de entrada não são enviados diretamente à rede neural, porque existe a necessidade de agrupar os dados a fim de efetuar pré - processamento para posterior processamento. São abordados os seguintes distúrbios neste trabalho: harmônicos, inter-hamônicos, sub-harmônicos, interrupção curta, notching, afundamento de tensão ( $\operatorname{sag}$ ), elevação de tensão (swell), spike e transitório oscilatório.

\section{MATERIAIS E MÉTODOS}

\subsection{Filtro $H P$}

O filtro Hodrick-Prescott, que é uma novidade deste trabalho do ponto de vista de técnicas para QEE, decompõe o sinal em duas componentes: tendência e cíclica. Em essência, removem-se as flutuações de baixa frequência dos sinais ou séries temporais. A hipótese é que, nessa separação, a variabilidade de baixa frequência representa a tendência de prazo mais longo, e a variabilidade de frequência mais alta representa os ciclos mais curtos e/ou aleatórios (Santana, 2017).

O filtro HP busca extrair a tendência, que é considerada estocástica, mas com variações suaves, ao longo do tempo e não correlacionadas com o ciclo, por meio da minimização com respeito a $\tau_{t}$ da seguinte expressão:

$$
j=\min \sum_{t=1}^{T} C_{t}^{2}+\lambda \sum_{t=3}^{T}\left(\Delta^{2} \tau_{t}\right)^{2}
$$

em que $c_{t} \triangleq y_{t}-\tau_{t}$ e $\Delta^{2}=(1-L)^{2}$, com L sendo o operador de defasagens, por exemplo $L x_{t}=x_{t}-1$.

Na equação (1), $T$ é a quantidade de amostras de dados em questão, e $\lambda$ é um parâmetro que penaliza a variabilidade da tendência. $\mathrm{O}$ parâmetro $\lambda$ é o parâmetro de suavidade com o qual se controla a aceleração da componente de tendência, isto é, as variações na taxa de crescimento da componente de tendência. O primeiro termo da equação (1) é a soma dos desvios da série com respeito à tendência ao quadrado e é uma medida do grau de ajuste. O segundo termo é a soma de quadrados das segundas diferenças das componentes de tendência e é uma medida do grau de suavidade.

\subsection{Discriminante Linear de Fisher}

Conforme Ferreira (2010) o discriminante linear de Fisher (FDR - Fisher Discriminat Ratio) é uma técnica bastante simples para discriminação de dados multidimensionais. Neste trabalho o FDR foi utilizado na etapa de préprocessamento como ferramenta de seleção de parâmetros, onde a função custo do critério FDR é:

$$
J_{C}=\left(m_{1}-m_{2}\right)^{2} \odot \frac{1}{D_{1}^{2}+D_{2}^{2}}
$$

onde $\mathrm{Jc}=\left[\mathrm{J}_{1} \cdots \mathrm{J}_{\mathrm{L}}\right]^{\mathrm{T}}, \mathrm{L}_{1}$ é o número total de parâmetros, $\mathrm{m}_{1} \mathrm{e}$ $\mathrm{m}_{2}$, e $\mathrm{D}_{1}^{2}$ e $\mathrm{D}_{2}^{2}$ são, respectivamente, os vetores de média e variância dos dados da classe 1 e da classe 2. O símbolo $\odot$ refere-se ao produto de Hadamard $r \odot s=r_{0} s_{0} \ldots\left[r L_{\tau}-1 s L_{\tau}-1\right]^{T}$.

Pode-se entender que os $i$-ésimos elementos do vetor de parâmetros, referentes aos maiores valores de $J_{c}$, representam os parâmetros que apresentam melhor separabilidade entre classes.

\subsection{Redes Neurais Artificiais (RNA)}

As redes neurais artificiais (RNA's) são sistemas de computação adaptativa inspirados nas características de processamento de informação encontrada nos neurônios reais e nas características de suas interconexões (Marques, et. al. 2010). A arquitetura de uma rede neural pode ser de diversas formas, conforme a necessidade da sua aplicação, estando diretamente ligadas com o algoritmo de aprendizagem usado para treinar a rede (Matsunaga, 2012).

A estrutura do neurônio de uma rede neural está diretamente ligada ao algoritmo de aprendizado a ser usado para treinar a rede. Neste trabalho, a arquitetura utilizada é do tipo "Redes Alimentadas Diretamente com Múltiplas Camadas". Esse tipo de arquitetura se distingue pela presença de uma ou mais camadas ocultas (ou intermediárias), cujos nós computacionais são chamados de neurônios ocultos ou unidades ocultas. A função dos neurônios ocultos é intervir entre a entrada externa e a saída da rede de uma maneira útil (Matsunaga, 2012; Bishop, 1995).

Tais redes são, normalmente, chamadas de perceptrons de múltiplas camadas (MLP - Multilayer Perceptron). Os perceptrons de múltiplas camadas têm sido aplicados com bastante sucesso para resolver diversos problemas complexos, através do seu treinamento de forma supervisionada com um algoritmo muito popular conhecido como algoritmo de retropropagação do erro (Ferreira, 2010).

\section{METODOLOGIA}

\subsection{Obtenção de Sinais para Análise}

Os sinais foram gerados computacionalmente, de forma sintética, pelo software MatLab ${ }^{\circledR} 2014$ (uso acadêmico individual), de acordo com a regulamentação do IEEE (IEEE, 2009). Todos os sinais gerados apresentam frequência fundamental igual a $60 \mathrm{~Hz}$, frequência do sistema elétrico brasileiro. A frequência de amostragem realizada foi de $15.360 \mathrm{~Hz}$. Desta forma, 256 amostras por ciclo são obtidas. Essa taxa de amostragem foi escolhida por ser suficiente para caracterizar a maioria dos distúrbios presentes nos sistemas elétricos.

O ruído branco foi definido de modo que atenda a relação sinal-ruído (SNR - signal-to-noise ratio) desejada: 


$$
S N R=20 \log \frac{A}{\sqrt{2 \sigma}}[d B]
$$

em que $A$ é a amplitude do sinal fundamental e $\sigma$ é o desvio padrão do ruído.

$\mathrm{Na}$ prática, sinais de tensão medidos nos sistemas elétricos possuem relação sinal ruído entre $50 \mathrm{~dB}$ e $70 \mathrm{~dB}$ (Josif; Miograd; Vladimir, 2007). Com a finalidade de analisar diferentes níveis de ruídos presentes no sinal, são utilizadas as relações sinal-ruído $20 \mathrm{~dB}, 30 \mathrm{~dB}, 40 \mathrm{~dB}, 50 \mathrm{~dB}, 60 \mathrm{~dB}$.

As características de cada evento de QEE, como amplitude e fase, são atribuídas de maneira aleatória em intervalos com distribuição uniforme. As amplitudes adotadas neste trabalho são normalizadas, ou seja, a tensão de pico nominal tem valor unitário. A fase é atribuída aleatoriamente, dentro do intervalo $[-\pi ; \pi]$ com distribuição uniforme.

Foram gerados 10.000 vetores de dados, onde o número de ciclos da senóide é de dez ciclos, dos quais: 1000 não possuem distúrbios; 1000 possuem distúrbio harmônico; 1000 possuem distúrbio inter-harmônico; 1000 possuem distúrbio sub-harmônico; 1000 possuem distúrbio interrupção curta; 1000 possuem distúrbio notching; 1000 possuem distúrbio afundamento de tensão; 1000 possuem distúrbio elevação de tensão; 1000 possuem distúrbio spike; 1000 possuem distúrbio transitório oscilatório

\subsection{Sistema de Monitoramento Proposto}

Uma das principais atribuições do monitoramento da qualidade de energia elétrica é a análise dos distúrbios elétricos. O sistema proposto, cujo diagrama é mostrado na Fig.1.

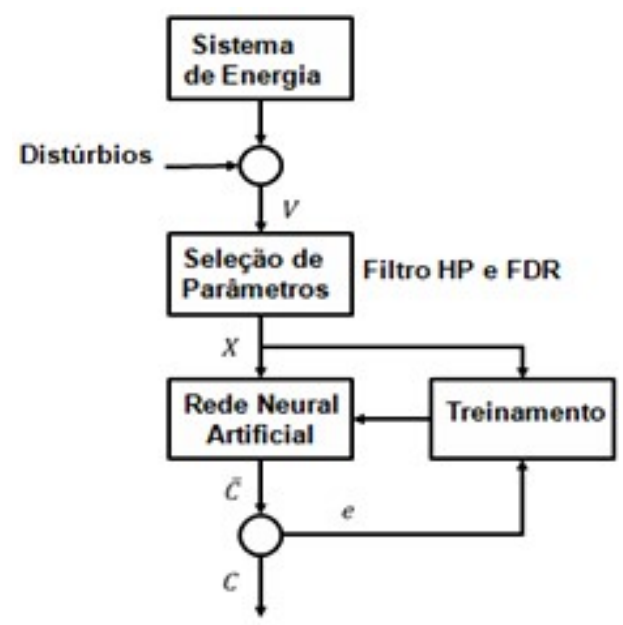

Fig.1 Sistema de Monitoramento Proposto.

O sistema de monitoramento é composto pelos estágios: geração dos sinais sintéticos, seleção de parâmetros (préprocessamento utilizando o filtro HP e o Discriminante de Fisher), rede neural MLP para detecção de distúrbios de qualidade de energia e treinamento da rede neural. A saída da rede será uma classe $C$, onde a classe zero será a do sinal sem distúrbio e a classe um a do sinal com distúrbio.

\subsection{Filtro Hodrick-Prescott}

O filtro Hodrick-Prescott é utilizado para separar o sinal elétrico em componente cíclica e de tendência, a fim de possibilitar a extração de características dos sinais. O filtro HP busca extrair a tendência do sinal, assim, o ruído presente no sinal (componente de alta frequência) é apresentado na componente cíclica, proporcionando análise livre de interferência do ruído quando se analisa a componente de tendência.

A Fig. 2 apresenta um sinal elétrico particular, o qual possui frequência fundamental de $60 \mathrm{~Hz}$, relação sinal-ruído de $20 \mathrm{~dB}$ e amplitude de $1 \mathrm{pu}$ (por unidade). No entanto, está poluído com um distúrbio do tipo transitório oscilatório, proveniente de chaveamento de bancos de capacitores.
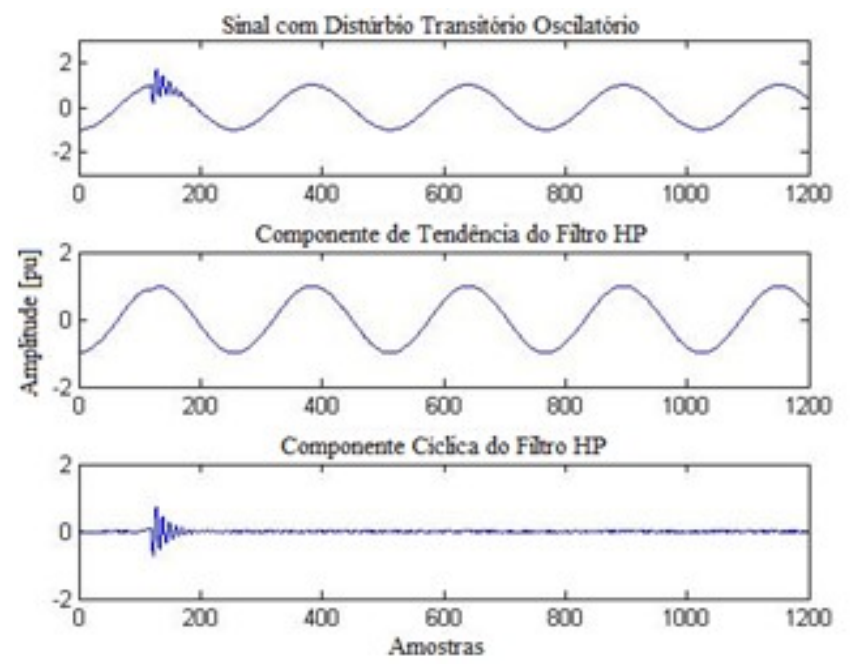

Fig.2 Filtro HP atuando em sinal com distúrbio Transitório Oscilatório.

Nota-se através da Fig.2 que o filtro HP decompôs o sinal original, em duas componentes: componente de tendência e componente cíclica. O ruído presente no sinal e o distúrbio transitório oscilatório, ambos componentes de alta frequência, apresentaram-se na componente cíclica. Enquanto o sinal livre de ruídos apresentou-se na componente de tendência, ou seja, o sinal de frequência fundamental em $60 \mathrm{~Hz}$ sem distúrbio e sem ruído.

\subsection{Fisher Discriminat Ratio}

O FDR, pela primeira vez considerado em conjunto com o Filtro HP, seleciona os parâmetros que apresentam uma melhor separabilidade entre classes distintas, verificando a distância entre as médias das classes, ponderada pelas suas variâncias. Dado um conjunto de dados, a variância é uma medida de dispersão que mostra o quão distante cada valor desse conjunto está do valor central, ou seja, médio. 
A Fig.3 referente ao evento notching, mostra graficamente o comportamento da função custo, como mostra a equação (2) para SNR de $50 \mathrm{~dB}$. Os parâmetros que são avaliados e selecionados através do FDR são descritos a seguir: Vx variância do sinal original; Vt - variância da componente de tendência; Vc - variância da componente de cíclica; Vrms valor eficaz do sinal original; Vmax - valor máximo do sinal original; Vmin - valor mínimo do sinal original; VrmsT valor eficaz da componente de tendência; VmaxT - valor máximo da componente de tendência; VminT - valor mínimo da componente de tendência; VrmsC - valor eficaz da componente cíclica; $\mathrm{VmaxC}$ - valor máximo da componente cíclica; VminC - valor mínimo da componente cíclica;

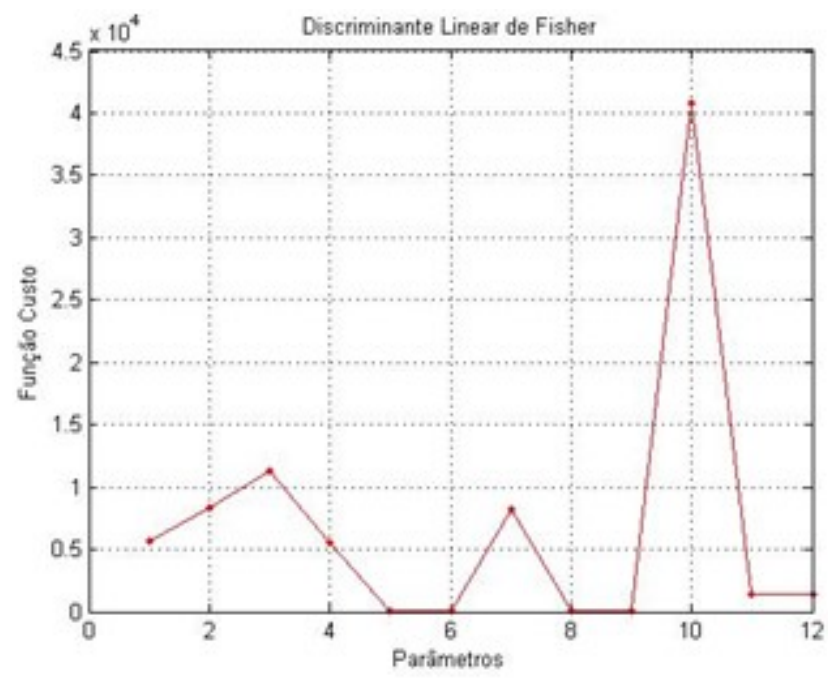

Fig.3 Gráfico da Função custo para notching de 50db com 2000 amostras.

Os parâmetros que apresentam uma melhor separabilidade entre classes distintas para o evento em destaque são os parâmetros 1, 2, 3, 7 e 10. Denotados por Vx, Vt, Vc, VrmsT e VrmsC, respectivamente.

\subsection{Rede Neural Multlayer Perceptron}

A topologia da rede MPL utilizada neste trabalho é apresentada na Fig.4. A rede neural foi implementada no software MatLab ${ }^{\circledR} 2014$ (uso acadêmico individual). São cinco parâmetros de entrada para cada distúrbio; quatro neurônios na camada escondida e um neurônio na camada de saída. A função de ativação utilizada foi à tangente hiperbólica e o algoritmo de treinamento utilizado foi backpropagation Levenberg-Marquardt. Foram definidas trinta épocas de treinamento e o desempenho como critérios de parada da rede.

O principal parâmetro de parada considerado foi o desempenho, tanto para os dados de treinamento quanto para os dados de teste. Quando ambos atingirem 100\%, o programa para de executar a apresenta os resultados. Caso não atingir $100 \%$, o programa vai executar todas as épocas de treinamento estabelecidas inicialmente. $\mathrm{O}$ valor zero na camada de saída representa a classe sem distúrbio e o valor um representa a classe com distúrbio. Os dados foram separados da seguinte maneira: $70 \%$ treinamento; $15 \%$ validação; $15 \%$ teste.

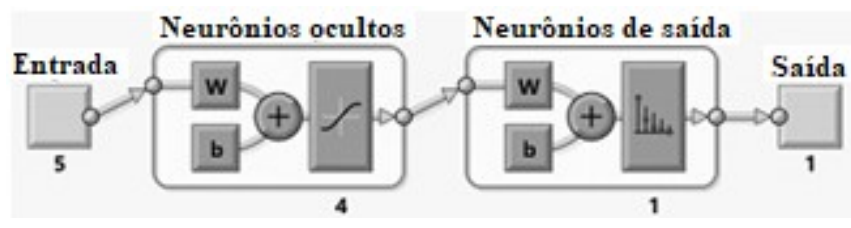

Fig.4 Topologia de rede MLP utilizada.

As chamadas amostras de treinamento servem para que as redes neurais "aprendam" e generalizem o conhecimento contido nas amostras, de forma a responder de forma adequada quando entradas não presentes no conjunto de treinamento forem apresentadas à rede. Dessa forma, mesmo que a informação fornecida esteja incompleta, ainda é possível obter-se um raciocínio correto. Enquanto os dados de validação e teste são utilizados para efetuar a classificação final à qual a rede neural está destinada.

\section{RESULTADOS E DISCUSSÃO}

Nesta seção, o sistema de detecção de distúrbios elétricos de QEE é apresentado. A detecção é implementada de maneira off-line, ou seja, apresenta uma análise de dados antes préprocessada, através de ferramentas matemáticas como o filtro HP e o FDR. Desta forma, há conhecimento dos dados a serem analisados, para que as técnicas de seleção de variáveis off-line sejam empregadas.

\subsection{Detecção de Distúrbios}

Neste item é analisado o desempenho de detecção utilizando a RNA. Os sinais analisados contêm dez ciclos da senoide, a fim de se verificar a robustez do sistema. A relação sinalruído tem variação entre $20 \mathrm{~dB}$ a $60 \mathrm{~dB}$. A detecção de distúrbios compreende basicamente a extração de parâmetros e a aplicação de um algoritmo que realize a detecção/classificação baseada nos parâmetros extraídos. A acurácia é utilizada como índice de desempenho do modelo proposto; e é calculada como se segue na equação (4):

$$
A_{C C}(\%)=\frac{\text { acertos }}{\text { acertos }+ \text { erros }} \cdot 100 \%
$$

onde os acertos são a somatória dos dados de saída classificados corretamente e os erros são a somatória dos dados de saída classificados de maneira incorreta.

A Tabela 1 mostra o desempenho de detecção $\left(\mathrm{A}_{\mathrm{cc}}\right)$, para os distúrbios considerados neste trabalho, em virtude da relação sinal-ruído. 
Tabela1 Tabela de deteç̧ão $\left(\mathrm{A}_{\mathrm{CC}}\right)$ de distúrbios elétricos.

\begin{tabular}{|c|c|c|c|c|c|}
\hline Eventos & 20dB & $\mathbf{3 0 d B}$ & $\mathbf{4 0 d B}$ & $\mathbf{5 0 d B}$ & $\mathbf{6 0 d B}$ \\
\hline Harmônico & 100 & 100 & 100 & 100 & 100 \\
\hline $\begin{array}{c}\text { Inter- } \\
\text { harmônico }\end{array}$ & 100 & 100 & 100 & 100 & 100 \\
\hline $\begin{array}{c}\text { Sub- } \\
\text { harmônico }\end{array}$ & 100 & 100 & 100 & 100 & 100 \\
\hline $\begin{array}{c}\text { Interrupção- } \\
\text { curta }\end{array}$ & 100 & 100 & 100 & 100 & 100 \\
\hline $\begin{array}{c}\text { Notching } \\
\text { Sag }\end{array}$ & 100 & 100 & 100 & 100 & 100 \\
\hline Swell & 97,8 & 97,8 & 98,8 & 98,4 & 99,4 \\
\hline Spike & 100 & 100 & 100 & 100 & 100 \\
\hline $\begin{array}{c}\text { Transitório } \\
\text { Oscilatório }\end{array}$ & 100 & 100 & 100 & 100 & 100 \\
\hline
\end{tabular}

Nota-se pela Tabela 1 que os melhores resultados foram para os distúrbios harmônico, inter-harmônico, sub-harmônico, interrupção curta, notching, spike e transitório oscilatório mostrando que os parâmetros utilizados, a partir do filtro HP e do FDR, são eficientes.

Para o distúrbio sag, o desempenho da rede não foi $100 \%$ para toda relação sinal-ruído, isto ocorre porque, se o afundamento for de muita intensidade, o distúrbio se confunde com uma interrupção curta e se o afundamento for de baixa intensidade, o distúrbio se confunde com um sinal sem distúrbio.

Percebe-se também que o distúrbio swell obteve um desempenho maior que $97 \%$ para toda relação sinal-ruído, podendo ser ocasionado ainda na etapa de processamento, na escolha dos parâmetros, enquanto os demais distúrbios obtiveram os melhores resultados.

\subsection{Comparação com Outros Métodos}

Nesta seção, o desempenho do filtro HP, é avaliado utilizando-se outros modelos de classificadores. São considerados dois métodos estatísticos: o modelo MLR (Multiple Linear Regression) e o SMV (Support Vector Machine). É utilizada a mesma base de dados que foi considerada para a RNA.

A Regressão Linear Múltipla é uma coleção de técnicas estatísticas para construir modelos que descrevem de maneira razoável relações entre várias variáveis explicativas de um determinado processo (Guarnieri, 2006). O SVM é uma técnica de aprendizado estatístico, baseada no princípio da Minimização do Risco Estrutural (SRM), que já foi bastante utilizada para resolver problemas de classificação (Cristianini; Shawe-Taylor, 1999).
A Tabela 2 apresenta o resultado comparativo do desempenho $\left(\mathrm{A}_{\mathrm{cc}}\right)$ entre os modelos considerados em relação aos valores amostrais obtidos experimentalmente para relação sinal-ruído de 50dB.

Tabela2 Resultado Comparativo entre Métodos.

\begin{tabular}{|c|c|c|c|}
\hline Eventos & MLP & MLR & SVM \\
\hline Harmônico & 100 & 100 & 100 \\
\hline Inter-harmônico & 100 & 98,75 & 99,9 \\
\hline Sub-harmônico & 100 & 99 & 100 \\
\hline Interrupção-curta & 100 & 100 & 100 \\
\hline Notching & 100 & 100 & 100 \\
\hline Sag & 100 & 92,7 & 99,5 \\
\hline Swell & 98,4 & 95,3 & 100 \\
\hline Spike & 100 & 100 & 100 \\
\hline Transitório Oscilatório & 100 & 100 & 100 \\
\hline
\end{tabular}

Percebe-se pela tabela 2 que os três classificadores apresentaram resultados bastante satisfatórios. A rede neural e o SVM tiveram desempenho superior da regressão linear múltipla quando se analisa os distúrbios sag e swell. Estes resultados evidenciam a eficácia do filtro proposto neste trabalho como ferramenta para extração de características de distúrbios de QEE.

\subsection{Matriz de Confusão}

A matriz de confusão é uma forma intuitiva de saber como seu classificador está se comportando. Ela oferece uma medida efetiva ao mostrar o número de classificações corretas entre detecção ou não do distúrbio em um sinal. $\mathrm{O}$ nome deriva do fato de que ela torna mais fácil ver se o sistema está confundindo as classes. Cada coluna da matriz representa as amostras de uma classe prevista, enquanto as linhas representam os casos de uma classe estimada pelo modelo. As entradas da matriz são representadas por $\mathrm{M}(\mathrm{Ci}$ ,$C j$ ), indicando o número de exemplos de $N$ que são da classe $C i$ mas que foram classificados pela hipótese $h$ como sendo da classe $C j$ como mostra a equação (5):

$$
M\left(C_{i}, C_{j}\right)=\sum_{\left\{\forall(x, y) \in N: y=C_{i}\right\}}\left\|h(x)=C_{j}\right\|
$$

onde o número de acertos, para cada classe, localiza-se na diagonal principal $\mathrm{M}(\mathrm{Ci}, \mathrm{Ci})$ da matriz; os demais elementos $M(C i, C j)$, para $i \neq j$, representam erros na classificação.

A matriz de confusão de um classificador ideal possui todos os elementos fora da diagonal principal iguais a zero uma vez que ele não comete erros. A matriz de confusão possibilita 
analisar a porcentagem de assiduidade das diversas amostras associadas a uma classe, assim como permite o entendimento e a quantificação das amostras classificadas de forma errada (Tan, et al. 2006).

Para todas as análises a seguir a matriz de confusão possui duas classes distintas, sendo a classe (0) ausência de distúrbio e a classe (1) presença de distúrbio. A Fig.5 apresenta a matriz de confusão que é utilizada, para avaliar o índice de acertos para detecção do distúrbio sag para relação sinalruído de $50 \mathrm{~dB}$ e 2000 amostras. Os modelos utilizados foram (a) MLR e (b) SVM.

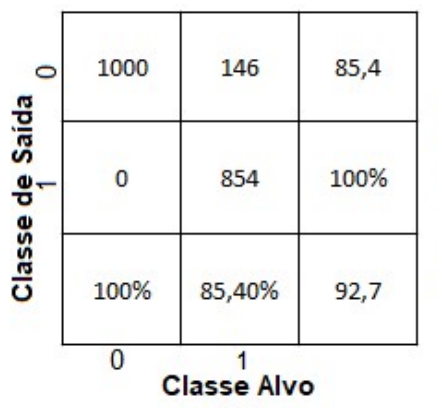

(a)

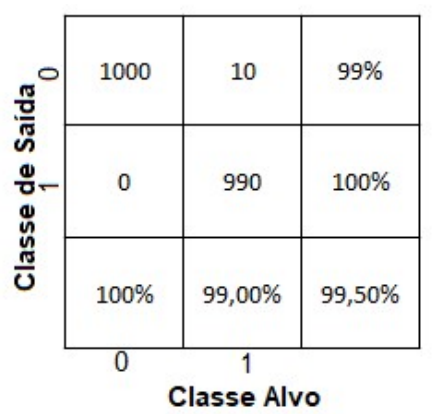

(b)
Fig.5 Matriz de Confusão para o distúrbio sag.

Observa-se que para o MLR em (a) das amostras sem distúrbio nenhuma amostra foi classificada como distúrbio, enquanto das 1000 amostras com distúrbio 146 amostras foram classificadas erradas como sendo sinal sem distúrbio, totalizando um índice de desempenho de $92,7 \%$ do modelo. Já para o SVM em (b) 10 amostras foram classificadas de maneira errada como sinal sem distúrbio, enquanto das 1000 amostras com distúrbios 990 foram classificadas certas como sendo sinal com distúrbio.

Os resultados obtidos mostram que o desempenho global das arquiteturas de MLP e do SVM alcançou um índice satisfatório no que diz respeito à precisão em comparação ao método MLR utilizado como mostrado na Tabela 2. Dessa forma, os três modelos apresentam bons resultados evidenciando dessa forma a eficiência do processamento dos dados e a eficácia do filtro HP utilizado nesse contexto.

\section{CONCLUSÕES}

São diversos os tipos de distúrbios de energia elétrica relacionados à qualidade da energia, os quais podem ser classificados em categorias que variam quanto ao efeito, duração e intensidade. $O$ fato é que esses distúrbios, quaisquer que sejam, precisam ser reconhecidos para que as contramedidas possam ser adotadas, e assim ocasionar uma melhora da qualidade de energia elétrica.
Neste contexto, este trabalho propôs duas abordagens que em conjunto se mostraram muito eficientes, sendo eles a utilização do Filtro HP e o FDR para seleção de parâmetros referente a cada distúrbio elétrico. $\mathrm{O}$ filtro HP foi considerado para a decomposição de sinais de tensão, em suas componentes cíclicas e de tendência, para posterior obtenção de atributos e análise por através das RNA's.

O desempenho de detecção foi bastante satisfatório para a RNA, bem como para os outros classificadores (MLR e SVM) utilizados na comparação. O filtro HP apresentou alto desempenho para baixo SNR, possibilitando uma análise eficiente com poucos ciclos da senóide e baixo custo computacional, ou seja, pouco tempo de processamento, enfatizando assim, a eficiência da etapa de préprocessamento do sistema de monitoramento de QEE. O tempo de processamento dos dados pela RNA e o MLR foi de $0,658 \mathrm{~ms}$ para todos os eventos, enquanto o SVM apresentou um tempo de processamento de 21 s para todos os eventos.

\section{REFERÊNCIAS}

Cristianini, N.; Shawe-Taylor, (1999). An introduction to support Vector Machines: and other kernel-based learning methods. New York: Cambridge University Press. 189p.

Bishop, C. Neural Networks for Pattern Recognition, Oxford: Oxford University Press. ISBN 0-19- 853849-9. 482 pages. 1995

Di Santo, K. G. et al, (2015). A review on smart grids and experiences in Brazil. Renewable and Sustainable Energy Reviews, London, v. 52, p. 1072-1082.

El-Hawary, M. E. (2014). The smart grid: state-of-the-art and future trends. Electric Power Systems Research, UK, v. 42 , n. $3 / 4$, p. $42-239$.

Ferreira, D. D. (2010). “Análise de Distúrbios Elétricos em Sistemas de Potência”. 2010. 233 p. Tese (Doutorado em Engenharia Elétrica) - Universidade Federal do Rio de Janeiro, Rio de Janeiro.

Guarnieri, R. (2006). Emprego de redes neurais artificiais e regressão linear múltipla no refinamento das previsões de radiação solar do modelo Eta. Insituto Nacional de Pesquisas Espaciais, $171 \mathrm{pp}$.

Institute of Electrical and Electronics Engineers - IEEE, (2009) "recommended practice for monitoring electric power quality". New York: IEEE. 91 p.

Instituto Nacional de Eficiência Energética - INEE, (2011). "A eficiência Energética e o Novo Modelo do setor Energético". Relatório Técnico Rio de Janeiro.

Josif, J. T, J.; Miograd. D. K and Vladimir. V. V, (2007). "A new power system digital harmonic analyzer". IEEE Transactions on Power Delivery, New York, v. 22, n. 2, p. 772-780.

Marques, C. A. G., Danton. D. F., Freitas, L. R., Duque, C. A., Ribeiro, M.V., (2010)." "Improved Disturbance Detection Technique for Power Quality Analysis", IEEE Transactions on Power Delivery Letters. (Aguardando Publicação)

Matsunaga, V. Y., (2012). Curso de Redes Neurais Utilizando Matlab. Apostila. Belém - Pará.

Santana, M. W. (2017). Agrupamento incremental de fluxo de dados para análise e monitoramento da qualidade de energia. 2017. 97 p. Dissertação (Mestrado em Engenharia de Controle e Automação)-Universidade Federal de Lavras, Lavras.

Tan, P.-N. et al, (2006) "Introduction to data mining". Noida: Pearson Education India.. 165 p. 